\title{
Detection of very small density fluctuations in pharmaceutical samples by application of the Modified Bronnikov Algorithm on micro-CT data
}

\author{
M. Boone, ${ }^{*}$ A. Almeida, ${ }^{* *}$ Y. De Witte, ${ }^{*}$ M. Dierick, ${ }^{*}$ J.P. Remon, ${ }^{* *}$ C. Vervaet, ${ }^{* *}$ and L. Van \\ Hoorebeke* \\ * Centre for X-ray Tomography (UGCT), Department of Subatomic and Radiation Physics, Ghent \\ University \\ ** Department of Pharmaceutical Sciences, Ghent University
}

Micro-CT $(\mu \mathrm{CT})$ has become a very useful tool in pharmaceutical sciences. It enables researchers to visualize and quantify porosity in a whole sample without destroying it, minimizing the risk of false results due to the sample treatment. For this reason, this powerful technique is often used for pore analysis [1]. Besides the porosity, density distribution of a pharmaceutical samples is also of interest, since it can give new insights in the behaviour of the sample. However, these density differences are often very small, and hardly visible when the CT data is noisy. This problem can be solved by exploiting the phase contribution in the X-ray projections. Since this phase signal is more sensitive to density variations than the absorption, it is ideal for the visualization of these structures.

In pure phase objects, i.e. samples that have no absorption, the phase signal can be reconstructed using the Bronnikov filter [2]. In reality, all objects have a certain absorption coefficient, making the algorithm incorrect. However, by adding a correction parameter in the filter, an approximation of the phase signal can be retrieved. This method is called the Modified Bronnikov Algorithm (MBA) [3] and can be used for a wide variety of objects[4].

A phase shift of the incoming X-ray beam causes refraction, which results in a typical black-white profile that can be seen in the X-ray projections. When such images are reconstructed with standard filtered back-projection, this intensity profile is also visible in the reconstructed slices as a very dense layer of the sample or feature, and a too low (or even negative) density just outside the feature. These densities are not physical and thus unwanted in tomographic reconstructions. One of the main advantages of the MBA is that it does not suffer from these phase artefacts. This improves the quality of the reconstructed cross sections and provides easier segmentation of the data, making it more suitable for porosity measurements, as can be seen in Figure 1.

The pharmaceutical sample under study is a hot stage extruded polymer/drug blend. After extrusion, a porous tablet is obtained, where the drug crystals sustain the polymer matrix with some large pores. During dissolution, the model drug is dissolved in water and released. The polymer matrix collapses due to a lack of structural strength of the material. This reduces the porosity and can be seen and measured with microCT. Besides the improved segmentation, application of the MBA on the sample showed features which could not be seen in the standard filtered backprojection (Fig. 2). MBA revealed denser regions in the tablet, which represent a change in polymer structure and density. This was confirmed by SEM analysis of the same sample, which showed regions void of micropores, thus resulting in a higher density, and possibly a structural change. Two images of these denser regions can be seen on Fig. 3. 
References

[1] D. Traini et al., Microscopy and Microanalysis 111 (2008) 13-15

[2] A.V. Bronnikov, Opt. Comm. 171 (1999) 239-244

[3] A. Groso et al., Opt. Express 14(18) (2006) 8103-8110

[4] M. Boone et al., Nucl. Instr. Meth. Phys. Res. B. accepted for publication
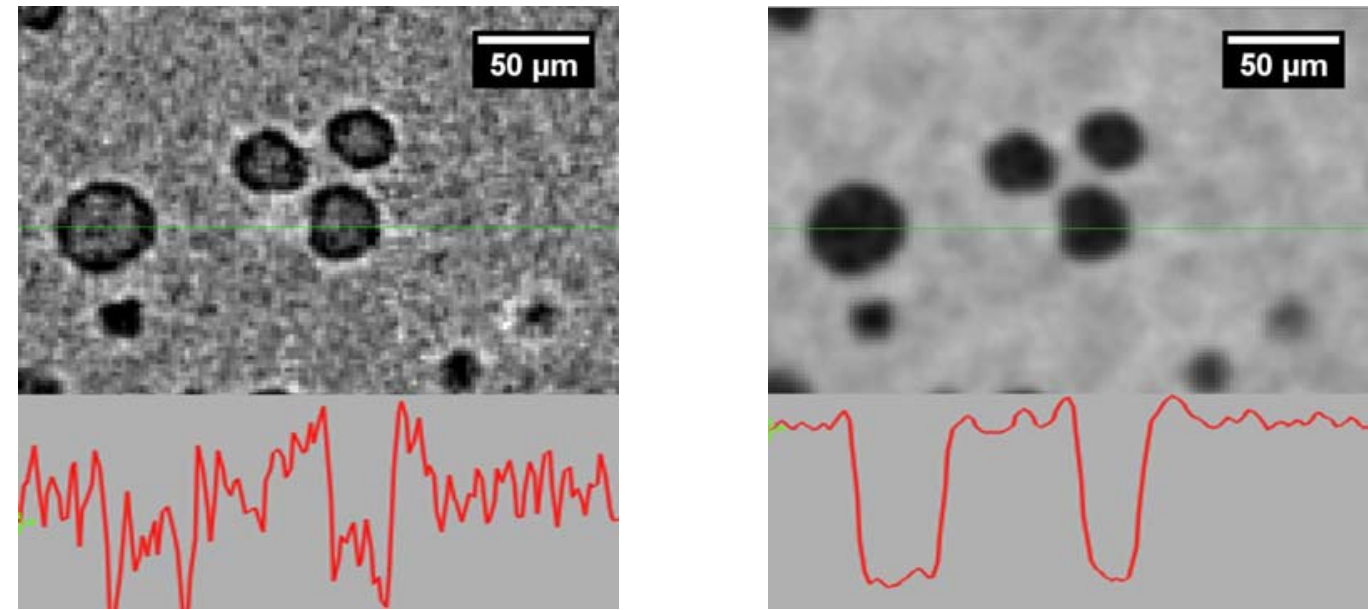

FIG. 1. Line profile on a standard filtered backprojection (left) and a MBA reconstructed slice (right).
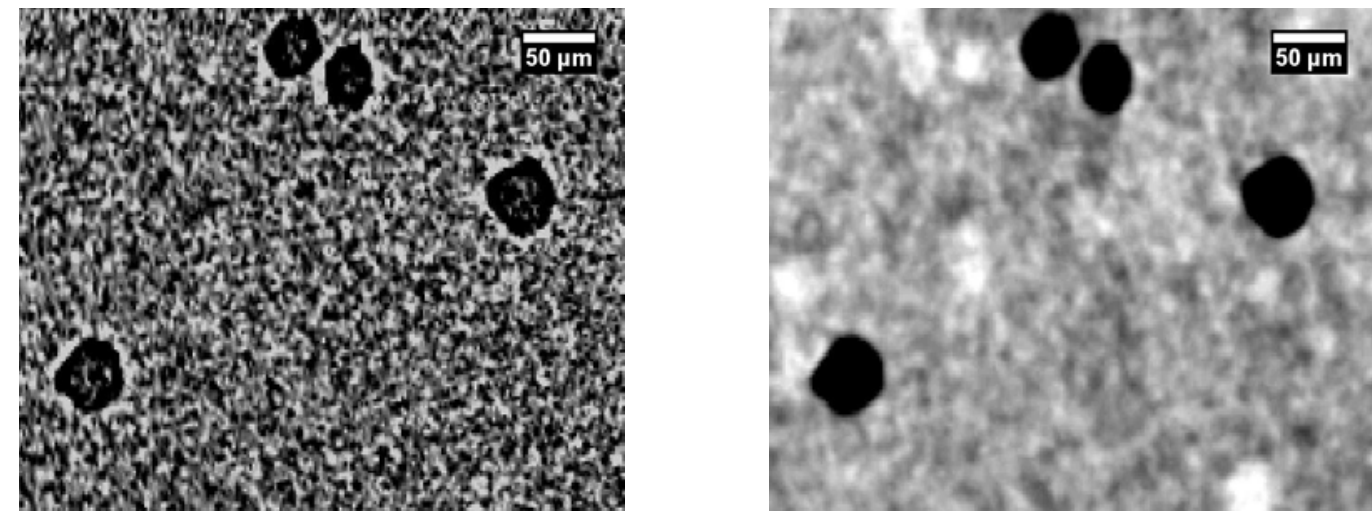

FIG. 2. FBP reconstructed image showing only pores (left) and MBA reconstructed slice showing both pores and dense regions (right).
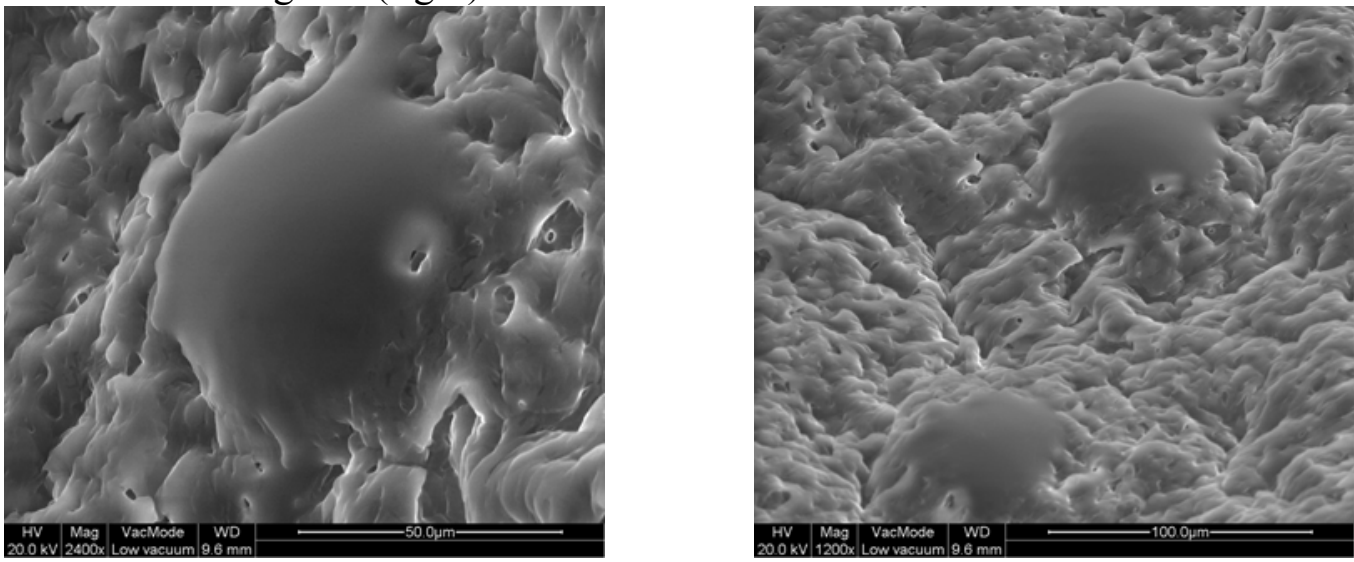

FIG. 1. Two views on the dense regions present in the sample after dissolution of the drug. 